\title{
Comparación de ecuaciones empíricas para el cálculo de la evapotranspiración de referencia en la Región del Libertador General Bernardo O'Higgins, Chile ${ }^{1}$
}

\author{
Marcela Sánchez Martínez² y Luis Carvacho Bart²
}

\begin{abstract}
RESUMEN
La evapotranspiración es un importante parámetro para diferentes campos científicos y su medida es necesaria para la adecuada gestión de los recursos hídricos, la construcción de obras de riego y la caracterización de los diferentes ambientes climáticos. Dada la complejidad de este fenómeno, se han elaborado numerosos métodos que permiten su estimación y que alcanzan una mayor o menor precisión y fiabilidad, comparados con mediciones realizadas en terreno. En este trabajo se comparan los resultados obtenidos con seis métodos empíricos comunes, de distintos requerimientos de datos meteorológicos y complejidad para su aplicación, para calcular la evapotranspiración de referencia, ETo. Se aplicaron en estaciones meteorológicas del área de estudio y para el periodo 1998-2006. Los resultados indican, en general, un buen grado de ajuste entre los diferentes modelos, aunque el de Ivanov se aleja de los demás en todos los observatorios situados en la costa o en zonas con influencia oceánica.
\end{abstract}

Palabras clave: Evapotranspiración de referencia, datos meteorológicos, métodos de estimación.

\begin{abstract}
Evapotranspiration is an important parameter for different scientific fields and its measurement is necessary for the proper management of hydric resources, the construction of irrigation infrastructure and the characterization of different climatic environments. Due the complexity of this process different methods have been developed in order to achieve its estimation, some of them more precise than others compared with direct measures in field. In this paper, the results obtained from six well known methods for estimating reference evapotranspiration (ETo) with different requirements of input data are compared. Data was obtained from meteorological stations in the study area for the period 1998-2006. The results show, in general, a good fit between the selected models, although the Ivanov model was not as precise as the other five ones, all of them in stations with oceanic and inner influence.
\end{abstract}

Key words: Reference evapotranspiration, meteorological data, estimation methods.

1 Proyecto FONDECYT 1085249. Artículo recibido el 24 de marzo de 2010, aceptado el 5 de abril de 2011 y corregido el 1 de agosto de 2011.
2 Instituto de Geografía, Pontificia Universidad Católica de Chile (Chile). E-mail: mism@uc.cl; Icarvach@ uc.cl 
La evapotranspiración, ET, como proceso resultante del desarrollo simultáneo de la evaporación y la transpiración vegetal requiere para su desarrollo de energía, que en último término procede del Sol. Interviene en el balance hidrológico y energético, asegurando el mantenimiento del ciclo hidrológico y que la energía solar recibida por la Tierra sea devuelta a la atmósfera. Se estima como promedio global que el $57 \%$ de la precipitación anual es transferida a la atmósfera a través de este proceso, pudiendo llegar a valores del $90 \%$ y $100 \%$ en zonas áridas o desérticas, respectivamente (Sánchez, 1992). Aparte de ser un parámetro de interés en la clasificación climática de los diferentes ambientes, al permitir la definición de periodos de déficit o exceso hídrico, es necesario conocer la ET, entendida esta como la demanda de agua que tienen los diferentes cultivos y plantaciones para un óptimo desarrollo agrícola o forestal. Esto permite una adecuada gestión de los recursos hídricos y económicos necesarios para la construcción de obras de irrigación y la planificación del riego (Valenzuela y Ferreira, 1985; Hargreaves, 1994; Comisión Nacional de Riego, 1997; Xu \& Singh, 2001; Droogers \& Allen, 2002; Temesgen et al., 2005; LópezMoreno et al., 2009), especialmente en las zonas mediterráneas, caracterizadas por una estación seca que coincide con la de mayores requerimientos de los cultivos.

La ET depende de numerosos factores meteorológicos, entre ellos la radiación solar como la fuente de energía fundamental para el desarrollo del proceso, la temperatura del aire como consecuencia de la anterior, la humedad relativa como medida de la capacidad evaporativa del ambiente y de la velocidad del viento que remueve constantemente el agua desde la superficie evaporante y transporta, en ocasiones, calor para mantener activo el proceso; pero, además, depende de las características de la vegetación del área, su tipo, densidad y estado de crecimiento, como también del suelo, sus propiedades y su contenido de humedad.

La evapotranspiración potencial, ETP, concepto introducido en 1948 por C. Thornthwaite, hace referencia a la máxima cantidad de agua que es capaz de transferir a la atmósfera una superficie de suelo totalmente cubierto de vegetación, que crece en condiciones óptimas y sin limitaciones en el suministro de agua, dependiendo solo de las condiciones atmosféricas prevalecientes en el momento en que se realiza la estimación. El concepto de ETP se hace más preciso al utilizar el de evapotranspiración del cultivo de referencia, ETo, introducido a fines de los años setenta y comienzos de los años ochenta del siglo pasado, con el fin de estudiar la demanda de evapotranspiración de la atmósfera, independientemente del tipo y desarrollo del cultivo (Allen et al., 1998). La ETo estima los efectos de las condiciones climáticas sobre la ET que presenta un tipo vegetal estándar tomado como referencia, habitualmente alfalfa o, más comúnmente, una gramínea, que crece disponiendo de toda el agua necesaria y libre de enfermedades (McKenney \& Rosenberg, 1993; Burman \& Pochop, 1994). En la actualidad el término evapotranspiración del cultivo de referencia ha sido reemplazado por el de evapotranspiración de referencia, queriendo indicar en ambos casos lo mismo. Aunque se reconoce que los conceptos de ETP y ETo, en estricto sentido, son diferentes, ambos indican cantidades máximas de agua perdida a favor del ambiente, y por ello en algunos estudios han sido considerados equivalentes (McKenney \& Rosenberg, 1993). Conociendo la ETo correspondiente a la superficie vegetal específica o estándar es posible definir coeficientes empíricos para calcular la ET correspondiente a otras cubiertas (Doorenbos y Pruitt, 1977; Allen et al., 1998). Estos coeficientes permiten dimensionar las demandas hídricas de una zona agrícola o forestal y programar debidamente el riego, consiguiéndose así que la evapotranspiración real o efectiva se iguale al máximo requerimiento de la vegetación, obteniendo los mejores rendimientos y haciendo un uso eficiente del agua.

Para conocer la magnitud de la ET se han propuesto desde comienzos del siglo XIX numerosos modelos denominados directos, teóricos o empíricos que pretenden calcular el agua transferida a la atmósfera en un área determinada, ya sea a través de mediciones directas del agua que entra a un área y la que regresa a la atmósfera, o a través de una estimación indirecta de esa cantidad. Estos modelos son diversos en cuanto a su complejidad, precisión y requerimientos de datos para su aplicación. Algunas características 
generales de ellos se presentan en Sánchez 2000 y 2001.

No obstante haberse comenzado a definir desde 1802 las primeras formulaciones teóricas, cuando Dalton enuncia su ley e introduce la ecuación del transporte de masa (Rosenberg et al., 1983; Fernández, 1995), no es sino es a partir de mediados del siglo XX que se inicia una profusa etapa de producción y aplicación de modelos empíricos basados en datos medidos en los observatorios meteorológicos, situación que se mantiene muy activamente hasta la actualidad en todo el mundo.

Los métodos empíricos se pueden dividir en tres grupos generales: aquellos basados en la temperatura del aire, en la radiación solar y temperatura, y aquellos basados en la ecuación de combinación del balance de energía y de la transferencia turbulenta del vapor de agua, también denominados semiempíricos (Sánchez, 1999). Las fórmulas del primer grupo son muy útiles cuando no se dispone de otros datos meteorológicos, pero entregan resultados menos confiables (McKenney \& Rosenberg, 1993) que los métodos de los otros grupos, aunque mejoran luego de realizar ajustes locales. Por su parte, los basados en la ecuación de combinación al tener, como los métodos teóricos, una base teórica más fuerte introducida en el modelo a través de coeficientes empíricos, pueden aplicarse en cualquier lugar del mundo y son los que entregan mejores resultados; no obstante, son altamente demandantes en datos de entrada.

Numerosos estudios se están realizando hoy para comparar los resultados que entregan estos métodos con diversos objetivos. En primer lugar, aparte de querer dimensionar la ET con la mayor precisión posible, los estudios se dirigen a determinar los métodos más fiables para un determinado ambiente climático, luego de ser contrastados sus resultados con las medidas directas tomadas en el terreno, con la evaporación de bandeja o con un método basado en principios físicos de reconocida precisión. De este tipo es el estudio de Xu \& Singh (1998) en Suiza; Xu \& Singh (2001) en Canadá; el de Suleiman \& Hoogenboom (2007) en Georgia, Estados Unidos; el de Pereira et al. (2009) en Brasil y el de Shi et al. (2008) en la provincia de Jilin en China.
De base física son la formulación de Penman (1948), los propuestos posteriormente a partir de él y, actualmente, el de Penman-Monteith FAO, recomendado por la Food and Agriculture Organization (FAO) para estudios en todo el mundo (Allen et al., 1998). Otras investigaciones, más que determinar el método más preciso, intentan conocer el grado de variación que presentan los resultados arrojados por las distintas ecuaciones, los cuales, normalmente tienen entre ellos el de Penman o Penman-Monteith FAO, como hacen Temesgen et al. (2005). Este último tipo de estudios tiene gran importancia, toda vez que hay una relación bastante directa entre la precisión de los métodos y la cantidad de variables y datos que requieren para su aplicación, datos que muy pocas veces están disponibles en su totalidad. Por esta razón aplican métodos de diversas exigencias, y consecuentemente de distinta precisión, y comparan los resultados para establecer el grado de diferencia entre los resultados y tenerlo en cuenta al momento de tomar decisiones. Otra alternativa, siguiendo las indicaciones dadas por Allen et al. (1998) en su Estudio FAO Riego y Drenaje $N^{\circ} 56$, es insistir en la aplicación de un modelo de altos requerimientos a partir de la estimación de las variables faltantes en función de las que se tienen. Una aplicación y evaluación de este tipo es la realizada por López-Moreno et al. (2009). Otros estudios, además de contrastar los resultados, ajustan algunos términos de las ecuaciones empíricas, luego de comparar los resultados dados por métodos de grandes y de bajos requerimientos; así se consigue adaptar los modelos a la realidad local, una mayor precisión de sus resultados y su replicación en espacios de similares características geográficas, pero con datos limitados o de dudosa calidad (Bautista et al., 2009; Droogers \& Allen, 2002; Xu \& Singh, 2001 y 2002; Vega y Jara (2009). Por último, se pueden señalar aquí también los estudios realizados con el objetivo de establecer la sensibilidad que tiene la ETP o ETo a la variación en la magnitud de alguna de las distintas variables meteorológicas que se requieren (Valenzuela y Ferreira, 1985). En algunos casos específicos estos cambios son atribuidos al cambio climático (McKenney \& Rosenberg, 1993). En estos trabajos se aplican diferentes métodos empíricos o de base física con los cuales se simulan y comparan los resultados, evaluando la sensibilidad de 
cada uno a los cambios que se estiman para distintos escenarios de cambio. Se documenta que los modelos de base física son más flexibles en este sentido y no dependen de las relaciones que pueden ser cambiantes a lo largo del tiempo (López-Moreno et al., 2009).

En este trabajo se comparan los resultados que entregan seis métodos empíricos de distintos requerimientos que han sido aplicados en estaciones meteorológicas que disponen de los datos necesarios, localizándose la mayoría de ellas en la Región del Libertador General Bernardo O'Higgins. Los métodos empleados son el de Ivanov, Hargreaves, Turc, Jensen-Haise, Penman y Priestley-Taylor. Más que centrarse en el comportamiento espacial y temporal del fenómeno en el área, se pone el acento en la comparación de los resultados que entregan estas diferentes formulaciones que modelan el proceso de ETo de distintos requerimientos.

En un primer apartado se reseña brevemente el área de estudio, luego se presenta la metodología donde se exponen los métodos seleccionados, para presentar luego los resultados y las consideraciones finales.

\section{Área y período de estudio}

La Región del Libertador General Bernardo O'Higgins se localiza en Chile, directamente al sur de la Región Metropolitana de Santiago y de la Región de Valparaíso. Se extiende, aproximadamente, entre los $33^{\circ} 50^{\prime}$ y los $34^{\circ} 45^{\prime}$ de latitud Sur, y desde la cordillera de los Andes en su límite con Argentina, por el este, hasta el océano Pacífico, por el oeste. Tiene una superficie de $16.387 \mathrm{~km}^{2}$ y se presentan en ella las cuatro grandes unidades morfológicas que caracterizan el territorio nacional sudamericano: de este a oeste la cordillera de los Andes, la depresión intermedia, la cordillera de la Costa y las planicies litorales, estas últimas de gran desarrollo en la región. Ambas cordilleras se presentan enérgicamente, acercándose en algunos sectores para dar lugar a la denominada cuenca de Rancagua, emplazada dentro de la depresión intermedia. Esta disposición del relieve y su situación con respecto al océano generan contrastes espaciales en los elementos naturales del paisaje, entre ellos, el clima, las características del suelo y la distribución de la vegetación. Dentro del clima templado general, el dominante en la región es del tipo mediterráneo con lluvias durante la estación fría y un periodo seco en la estación cálida; esta última, conforme el Atlas Agroclimático de Chile (INIA, 1989), alcanza entre cuatro y seis meses, según la estación meteorológica considerada. Sin embargo, en la alta cordillera es posible identificar un clima frío provocado fundamentalmente por la altura.

El período de estudio corresponde a los meses comprendidos entre septiembre de 1998 y diciembre de 2006, esta extensión, junto a las condiciones antes señaladas, hacen de la región un área adecuada para realizar un estudio de estas características, pues presenta una variabilidad temporal y espacial del fenómeno que favorece el contraste de resultados.

\section{Metodología}

\section{Datos utilizados}

Se utilizan en el estudio datos diarios de doce estaciones meteorológicas (de 21 seleccionadas previamente), nueve de ellas se localizan en la Región del Libertador General Bernardo O'Higgins, una en la costa de la Región de Valparaíso, una en la Región Metropolitana de Santiago y una en la depresión intermedia de la Región del Maule. La localización de los observatorios se presenta en la Figura $N^{0} 1$. Como se puede ver en ella, la distribución tiene defectos, pues se presentan áreas, en términos relativos, bien cubiertas y otras en que hay ausencia de ellas. Estos observatorios son administrados por la Dirección Meteorológica de Chile y son de carácter agrometeorológico. Entre las variables necesarias para este estudio que registran, están temperatura máxima, mínima, media; humedad relativa a las 12,18 y 00 horas, o media diaria; horas de sol o nubosidad a las 12, 18 y 00 horas, o ambas (prácticamente no se registra radiación solar directa que es la variable ideal para estos estudios), y recorrido del viento diario. Aunque hay otros organismos públicos y privados que también tienen registros, se consideraron solo estas estaciones, pues emplean instrumentos estandarizados, lo que garantiza el uso de datos 
comparables. Lamentablemente, los registros adquiridos presentan numerosas carencias de datos, a veces por periodos prolongados de varios meses. En primer lugar, se realizó un análisis exploratorio de ellos para detectar datos erróneos, faltantes o mal registrados, y luego se procedió a completar algunas series tomando en consideración las indicaciones dadas por Allen et al. (1998). Se hizo un análisis de correlación entre estaciones próximas y situadas en espacios de similares características geográficas para completar por regresión los datos faltantes. No obstante, por carencias simultáneas entre ellas no fue posible completar las series, situación negativa que se une a la defectuosa distribución.

\section{Métodos empleados}

En este trabajo se emplean métodos empíricos correspondientes a los tres grupos generales, algunos de ellos considerados en un estudio realizado por los autores con anterioridad (Sánchez y Carvacho, 2005). Son métodos de amplia difusión, muy utilizados en todo el mundo y algunos en Chile también. Han sido elaborados, unos para estimar la ETP y otros directamente para un cultivo de referencia, ETo; no obstante, conforme a diversos autores y publicaciones, y en estudios como el que se presenta, esta distinción se ha hecho equivalente (Jensen et al., 1990; McKenney \& Rosenberg, 1993; Xu \& Singh, 2001; Xu \& Singh, 2002; Douglas et al., 2009; Bautista et al., 2009; Vega y Jara, 2009). Por otra parte, aunque su uso se ha realizado, mayormente con datos promedios mensuales a partir de registros de varios años, como en algunos estudios hechos en Chile, de igual forma pueden aplicarse a datos correspondientes a un año en particular, promedios mensuales, decadales e incluso diarios. Se presentan en los apartados siguientes.

Figura $\mathrm{N}^{0} 1$

Localización de las estaciones meteorológicas utilizadas

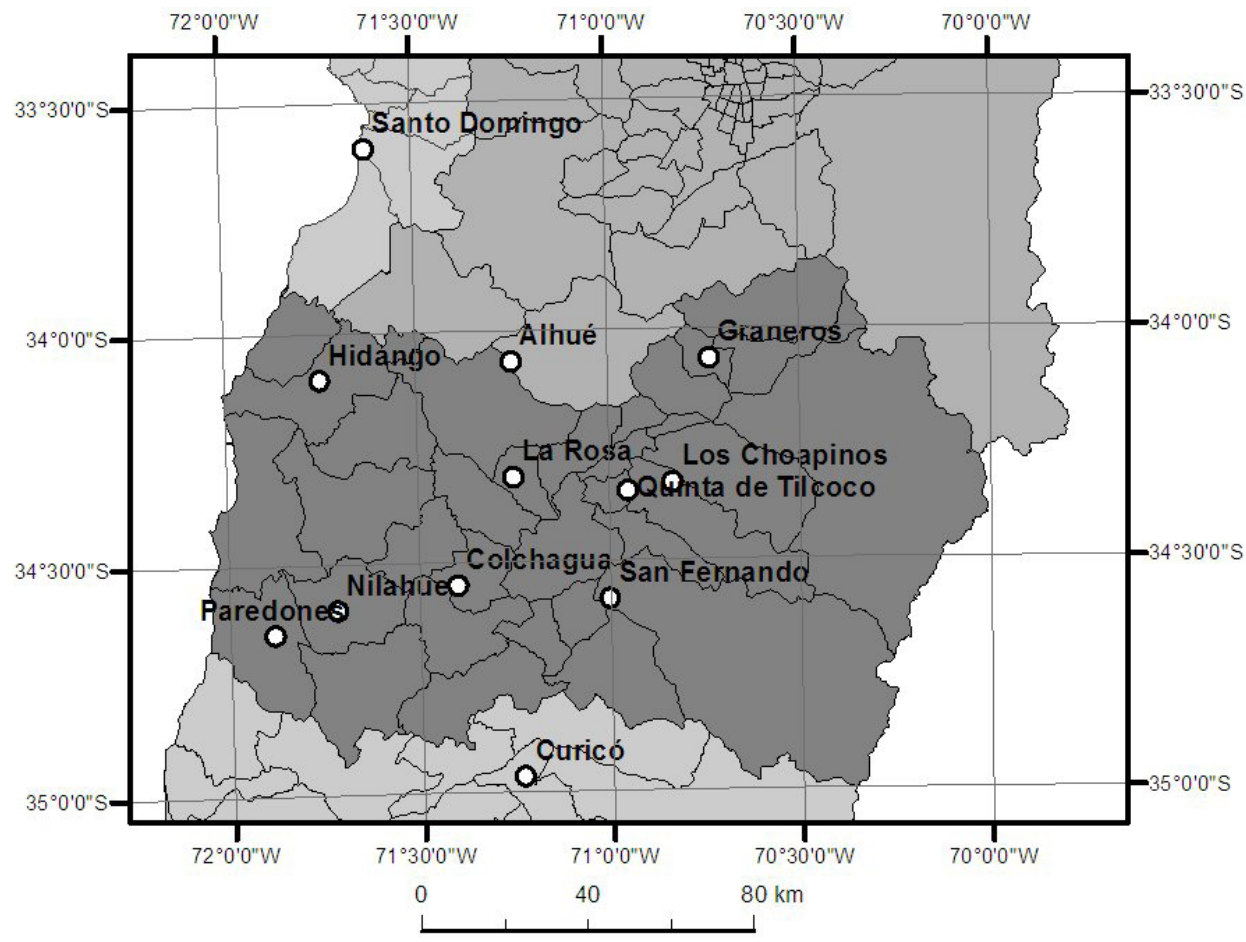

Fuente: Elaboración propia. 


\section{Método de Ivanov}

Este modelo fue propuesto por el investigador ruso Konstantin Evgenevich Ivanov en 1954. Está basado en la temperatura del aire y la humedad relativa. Ha sido aplicado en Chile por Santibáñez et al. (1979), Merlet (1986), Merlet y Santibáñez (1989) y por la Comisión Nacional de Riego (1997). Su fórmula, conforme a la última referencia, se escribe como:

$$
E T P=0,018 \cdot\left(25+T_{m}\right)^{2} \cdot(100-H R)
$$

Donde:

$E T P=$ evapotranspiración potencial, expresada en mm/día;

$T_{m}=$ temperatura media del aire, expresada en ${ }^{\circ} \mathrm{C}$;

$H R=$ humedad relativa del aire, expresada en porcentaje.

Buenos ajustes de este método en comparación con otros que calculan la ETo, que también se usan en este estudio, fueron reportados por Chemin (2003) al aplicarse a datos decadales en Uzbekistán.

\section{Método de Hargreaves}

La ecuación de Hargreaves, propuesta por Hargreaves y otros en 1985 y tomada de Hargreaves (1994), se escribe como:

$$
\mathrm{ET}_{0}=0,0023 \cdot \mathrm{R}_{\mathrm{a}} \cdot\left(T_{m}+17,8\right) \cdot\left(\mathrm{T}_{\text {máx }}-\mathrm{T}_{\text {min }}\right)^{1 / 2}
$$

Donde:

$E T o$ = evapotranspiración de referencia, expresada en $\mathrm{mm} / \mathrm{día}$;

$R_{a}=$ radiación solar extraterrestre, expresada en $\mathrm{mm} /$ día;

$T_{m}=$ temperatura media diaria, entendida como el promedio de la temperatura máxima y la temperatura mínima del periodo, expresada en ${ }^{\circ} \mathrm{C}$;

$T_{\text {máx }}=$ temperatura máxima diaria, expresada en ${ }^{\circ} \mathrm{C}$;

$T_{\text {mín }}=$ temperatura mínima diaria, expresada en ${ }^{\circ} \mathrm{C}$.

Este método empírico incorpora el término de radiación solar extraterrestre, es decir, la radiación solar recibida sobre una superficie horizontal en el límite exterior de la atmósfera, la cual puede calcularse fácilmente o tomar su valor desde tablas ya elaboradas, dado que es función de la latitud del lugar y de la época del año. No obstante lo anterior, los parámetros fundamentales siguen siendo los relativos al estado térmico del aire. Este método permite estimaciones de ETo y se puede aplicar a períodos diarios. Es uno de los más simples y más precisos métodos empíricos (Jensen et al., 1997), por lo que su uso es recomendado en situaciones donde no es posible aplicar otros y evitar así la confusión causada por la gran diversidad de métodos de este tipo (Hargreaves, G.H., 1994; Droogers \& Allen, 2002). Referencias sobre su uso en Chile, aunque con formulaciones diferentes, se indican en Sánchez (2001). Fue utilizado, junto a otros seis métodos por $\mathrm{Xu}$ \& Singh (2001) en Canadá; por Temesgen et al. (2005) en California, en un estudio similar al que aquí se presenta; por Hargreaves (1994), donde lo compara con resultados obtenidos por otras ecuaciones y mediciones lisimétricas de distintos lugares de Europa, Estados Unidos y Australia; por Vega y Jara (2009) en ambientes climáticos diferentes en Ecuador.

\section{Método de Turc}

Este método propuesto por L. Turc en 1961 se basa sobre la temperatura del aire y la radiación solar recibida en la superficie, principal fuente de energía que alimenta el proceso de evapotranspiración, por ello se considera de mayor precisión que los anteriores. Es uno de los métodos clásicos de mayor uso en el mundo debido a sus buenos resultados. En Chile se ha utilizado en numerosos estudios (Sánchez, 2001) cuando no ha sido posible aplicar el método de Penman que se presentará enseguida, o sus derivaciones, y cuando hay disponibles datos de radiación global, muy escasa en los observatorios chilenos. No obstante, este parámetro puede estimarse a partir de datos más comunes como horas de sol diarias o nubosidad, procedimiento que se ha seguido aquí, atendiendo a las indicaciones dadas por Burman y Pochop (1994).

El método de Turc, tomado de Comisión Nacional de Riego (1997), se expresa como:

$\mathrm{ETP}=0,013 \times\left(\mathrm{R}_{\mathrm{S}}+50\right) \times\left(\frac{\mathrm{T}_{\mathrm{m}}}{\mathrm{T}_{\mathrm{m}}+15}\right) \times 1+\left(\frac{65-\mathrm{HR}}{120}\right)$ 
Si la humedad relativa media del período es mayor a $65 \%$, y, como sigue, si la humedad relativa media del período es menor a $65 \%$ :

$E T P=0,013 \times\left(R_{S}+50\right) \times\left(\frac{T_{m}}{T_{m}+15}\right) \times 1+\left(\frac{65-H R}{120}\right)$

Donde:

$E T P=$ evapotranspiración potencial, expresada en mm/día;

$R_{S}=$ radiación global incidente, expresada en $\mathrm{cal} / \mathrm{cm}^{2} /$ día;

$T_{m}=$ temperatura media del aire, expresada en ${ }^{\circ} \mathrm{C}$;

$H R=$ humedad relativa media del período.

\section{Método de Jensen-Haise}

El método propuesto por M.E. Jensen y H.R. Haise, en 1963, también basado sobre la radiación solar y la temperatura del aire, tomado de Rosenberg et al. (1983), se presenta como:

$$
E T_{0}=R_{S} \times\left(0,025 * T_{m}+0,08\right)
$$

Donde:

$E T O=$ evapotranspiración de referencia, expresada en $\mathrm{mm} / \mathrm{día}$;

$R_{S}=$ radiación global incidente, expresada en $\mathrm{mm} /$ día;

$T_{m}=$ temperatura media diaria, expresada en ${ }^{\circ} \mathrm{C}$.

En Chile ha sido aplicado en distintos estudios señalados en Sánchez (2001), aunque algunos emplean formulaciones diferentes. La ecuación presentada fue utilizada por Pereira et al. (2009) en Brasil junto a otros nueve métodos que se comparan con la ecuación de base física de Penman-Monteith FAO, tomada como referencia de mayor precisión. Jabulani (2008) utiliza este método y obtiene para cada mes ecuaciones calibradas localmente para estimar la ETo en Zimbabwe.

\section{Método de Penman}

En su formulación original de 1948 el método del investigador inglés H.L. Penman constituye la primera expresión de base física para calcular la evapotranspiración potencial y que aplica la ecuación de combinación del balance de energía y de la transferencia turbulenta del vapor de agua (Rosenberg et al., 1983; Sánchez, 1992; Burman \& Pochop, 1994), por ello presenta un término radiativo y otro aerodinámico. El modelo está fundado sobre el cálculo previo de la evaporación potencial de una cubierta de agua libre, Eo, el cual es transformado luego en evapotranspiración de una superficie cubierta con vegetación, ETo, con el uso de un coeficiente de reducción, $f$, variable y obtenido empíricamente. El método de Penman, tomado de Sánchez (1992) se expresa como:

$E T P=f \times E_{0}$

$E_{0}=\left(\frac{\Delta}{\Delta+\gamma}\right) \times\left(\frac{R_{n}-6}{\lambda}\right)+\left(\frac{\gamma}{\Delta+\gamma}\right) \times f u_{2} \times\left(e_{s}-e_{a}\right)$

Donde:

$E T P=$ evapotranspiración de referencia, expresada en $\mathrm{mm} /$ día;

$f=$ factor empírico, variable según el mes;

Eo = evaporación de una superficie de agua libre, expresada en $\mathrm{mm} /$ día;

$\Delta=$ pendiente de la curva de saturación de vapor/ temperatura, expresada en $\mathrm{kPa} /{ }^{\circ} \mathrm{C}$;

$\gamma=$ constante psicrométrica, expresada en $\mathrm{kPa} /{ }^{\circ} \mathrm{C}$;

$R_{n}=$ radiación neta, expresada en $\mathrm{MJ} / \mathrm{m}^{2} /$ día;

$G=$ flujo de calor del suelo, expresada en $\mathrm{MJ} /$ $\mathrm{m}^{2}$ /día;

$\lambda=$ calor latente de vaporización, expresado en $\mathrm{MJ} / \mathrm{kg}$;

$\mathrm{fu}_{2}=$ función del viento, definida como: siendo $U_{2}$ el recorrido del viento diario medido a 2 metros, expresado en km/día;

$\left(e_{s}-e_{a}\right)=$ déficit de presión de vapor, expresado en $\mathrm{kP3}, 5 \cdot\left(10+0,009 \cdot U_{2}\right) \mathrm{a}$

Aunque tiene como limitación la dificultad de disponer de toda la información requerida, básicamente en lo que se refiere a medidas de radiación y de viento, y de haberse formulado hace poco más de sesenta años, gracias a su fundamento en principios físicos este método puede usarse sin restricciones en cualquier ambiente climático. Es uno de los más completos y de los que da resultados más ajustados a la realidad bajo condiciones climáticas muy diversas. Fue el método recomendado por FAO y considerado parámetro con el cual contrastar resultados obtenidos con otros métodos. A partir de él, y manteniendo las mismas ventajas, se elaboraron numerosos modelos, entre ellos el de Penman-Monteith, que contiene las modifi- 
caciones hechas por J.L. Monteith en 1965. En la actualidad FAO (Allen et al., 1998), la American Society of Civil Engineers (ASCE) y la International Commission of Irrigation (ICID) recomiendan utilizar el método Penman-Monteith-FAO para calcular la ETo. Estos últimos tres métodos fueron utilizados por los autores de este trabajo en una investigación anterior en la que se obtuvieron resultados similares entre ellos (Sánchez y Carvacho, 2005).

\section{Método de Priestley-Taylor}

El modelo propuesto en 1972 por G.H.B. Priestley y R.J. Taylor corresponde a un método de combinación simplificado, pues el término relacionado con el déficit de presión de vapor, presente en todos los modelos de combinación, se encuentra aquí también, aunque reemplazado por un coeficiente empírico equivalente (Jensen et al. 1990). Conforme a Jensen et al. (1990), la ecuación adquiere la siguiente forma:

$$
E T_{0}=1,26 \times\left(\frac{\Delta}{\Delta+\gamma}\right) \times\left(R_{n}-G\right)
$$

Donde:

$E T o=$ evapotranspiración del cultivo de referencia, expresada en $\mathrm{mm}$ /día;

= pendiente de la curva de saturación de vapor/temperatura, expresada en $\mathrm{kPa} /{ }^{\circ} \mathrm{C}$;

= constante psicrométrica, expresada en $\mathrm{kPa} /$ ${ }^{\circ} \mathrm{C}$;

$R_{n}=$ radiación neta, expresada en $\mathrm{mm} /$ día;

$G=$ flujo de calor del suelo, expresada en $\mathrm{mm} /$ día.

Según antecedentes proporcionados por Sánchez (1992) este método y el de Penman arrojan resultados muy similares. Por esta razón y su simplicidad es bastante utilizado (Suleiman y Hoogenboom, 2007). Puede ser aplicado para obtener estimaciones para períodos diarios (McKenney \& Rosenberg, 1993).

\section{Análisis realizados}

Se aplicaron las fórmulas seleccionadas y se obtuvieron los resultados promedio de ETo para los 300 promedios decadales del periodo, en las 12 estaciones con las variables necesarias y para cada uno de los métodos presentados. Cabe recordar que se obtuvieron series temporales incompletas, pues había carencia de datos básicos para periodos de distinta longitud en las diferentes estaciones. En los observatorios que tenían registros de todos los datos meteorológicos básicos en el periodo se contó, inicialmente, con 3.044 series de datos diarios. Una vez obtenidos los resultados iniciales, y teniendo en cuenta que el estudio no pretende determinar el método más fiable, sino el grado de similitud o diferencia entre los resultados obtenidos, se procedió a realizar análisis de correlación entre los métodos en cada estación, tal como ha sido realizado en algunos trabajos consultados para explicar las tendencias en las estimaciones de modelos predictivos. Se contrastaron las matrices de correlación obtenidas, especialmente las correspondientes a estaciones situadas en ambientes climáticos diferentes. Por otra parte, se hicieron comparaciones gráficas de los resultados, revisando la magnitud y evolución temporal de la ETo estimada con los distintos métodos. Las diferencias entre ellos se dimensionaron a través del cálculo del error cuadrático medio entre cada uno comparado con el promedio de los valores, establecido como patrón. Se realizó un análisis de regresión entre cada uno de los métodos y el valor de referencia para observar el comportamiento de los valores obtenidos, en tanto la potencial sobrestimación o subestimación de acuerdo a la magnitud de los valores de ETo. Finalmente, se realizó un análisis de varianza (Ebdon, 1982) para determinar la existencia de diferencias significativas entre los valores de ETo alcanzados por los métodos empleados y por estación meteorológica.

\section{Resultados}

En cada estación meteorológica se realizó un análisis de correlación con los resultados decadales obtenidos con cada uno de los seis métodos empleados. Este análisis demostró buenas y muy buenas tendencias entre todos ellos. Los coeficientes de correlación oscilaron entre un valor mínimo de 0,749 , medido entre los métodos de Ivanov y de PriestleyTaylor en la estación de Santo Domingo (presentándose el segundo valor más bajo entre los mismos métodos en Paredones), y máximos sobre 0,99 entre los métodos de Turc y de Jensen-Haise, entre Penman y Priestley- 
Taylor, y entre Turc y Penman en nueve estaciones. Considerando todas las estaciones, las correlaciones más bajas se dan entre el método de Ivanov y los restantes; no así entre los otros métodos donde, en todos los casos, superan un valor de 0,978. Esta situación relativa se mantiene en todas las estaciones, aunque se puede establecer una diferencia entre las estaciones señaladas como de secano costero y secano de interior, y las situadas en la depresión intermedia (distinción hecha por la Dirección Meteorológica de Chile en su catastro de estaciones), pues en estas últimas las correlaciones con el método de Ivanov son mejores. Los buenos resultados entre los métodos de Penman y de Turc, a diferencia de lo que ocurre entre estos y el de Ivanov, son consistentes con lo presentado por Merlet y Santibáñez (1989) y por la Comisión Nacional de Riego (1997). En los Cuadros $\mathrm{N}^{\circ} 1$ y $\mathrm{N}^{\circ} 2$ se muestran las matrices de correlación correspondientes a las estaciones de Paredones y de Quinta de Tilcoco, representativas, respectivamente, de los ambientes secano interior y de la depresión intermedia.

Si bien entre los métodos se advierten buenas y muy buenas correlaciones o tendencias en el cálculo de la ETo, estas no muestran las variaciones que aquellos reproducen en la magnitud de la ETo. Las Figuras $N^{\circ} 2$ y $\mathrm{N}^{\circ} 3$ muestran en las estaciones de Paredones y Quinta de Tilcoco, respectivamente, la ETo calculada con los seis métodos para los 300

\section{Cuadro $N^{\circ} 1$}

Matriz de correlación entre los seis métodos seleccionados para estimar la ETo.

Estación Paredones (latitud 34 39' S; longitud 71 53’ W; altitud 125 m)

\begin{tabular}{|l|c|c|c|c|c|c|}
\hline \multicolumn{1}{|c|}{ Métodos } & Ivanov_Pa & Hargreaves_Pa & Turc_Pa & Jensen-Haise_Pa & Penman_Pa & Priestley-Taylor_Pa \\
\hline Ivanov_Pa & 1 & 0,827 & 0,816 & 0,830 & 0,815 & 0,771 \\
Hargreaves_Pa & 0,827 & 1 & 0,978 & 0,980 & 0,984 & 0,980 \\
Turc_Pa & 0,816 & 0,978 & 1 & 0,996 & 0,995 & 0,993 \\
Jensen-Haise_Pa & 0,830 & 0,980 & 0,996 & 1 & 0,992 & 0,989 \\
Penman_Pa & 0,815 & 0,984 & 0,995 & 0,992 & 1 & 0,997 \\
Priestley-Taylor_Pa & 0,771 & 0,980 & 0,993 & 0,989 & 0,997 & 1 \\
\hline
\end{tabular}

Fuente: Elaboración propia.

\section{Cuadro $\mathrm{N}^{\circ} 2$}

Matriz de correlación entre los seis métodos seleccionados para estimar la ETo. Estación Quinta de Tilcoco (latitud 34 $21^{\prime}$ S; longitud $70^{\circ} 57^{\prime} \mathrm{W}$; altitud $265 \mathrm{~m}$ )

\begin{tabular}{|l|c|c|c|c|c|c|}
\hline \multicolumn{1}{|c|}{ Métodos } & Ivanov_QT & Hargreaves_QT & Turc_QT & Jensen-Haise_QT & Penman_QT & Priestley-Taylor_QT \\
\hline Ivanov_QT & 1 & 0,956 & 0,959 & 0,957 & 0,960 & 0,943 \\
Hargreaves_QT & 0,956 & 1 & 0,983 & 0,979 & 0,990 & 0,987 \\
Turc_QT & 0,959 & 0,983 & 1 & 0,998 & 0,997 & 0,996 \\
Jensen-Haise_QT & 0,957 & 0,979 & 0,998 & 1 & 0,996 & 0,995 \\
Penman_QT & 0,960 & 0,990 & 0,997 & 0,996 & 1 & 0,998 \\
Priestley-Taylor_QT & 0,943 & 0,987 & 0,996 & 0,995 & 0,998 & 1 \\
\hline
\end{tabular}

Fuente: Elaboración propia. 
valores decadales del periodo estudiado. La evolución sinusoidal del fenómeno a lo largo del año y los mayores valores de ETo en las estaciones del interior son reproducidos por todos los modelos; no obstante, el de Ivanov entrega los valores más alejados de los otros en todas las estaciones, especialmente en aquellas localizadas en la zona del secano costero o en la zona costera propiamente. En el otro extremo, Penman y Turc proporcionan los más próximos entre sí, a los que se puede unir también el de Priestley-Taylor. En términos generales, y precisando más la situación, en la estación cálida los valores más altos de ETo son entregados por Jensen-Haise y los más bajos por Ivanov; por su parte, en la estación fría los entregan los métodos de Hargreaves y de Priestley-Taylor, respectivamente. Conforme a los resultados en estas estaciones, se puede decir que los métodos basados en la radiación solar y en la ecuación de combinación son, en general, los más consistentes y ajustados entre sí.

Para dimensionar la magnitud de la variación de la ETo estimada con los diferentes modelos en términos absolutos se calculó en cada estación meteorológica el error cuadrático medio (expresado en $\mathrm{mm} / \mathrm{d}$ ía) de cada método en relación al valor estándar establecido, en este caso, el promedio de los seis valores obtenidos. En la Figura $\mathrm{N}^{\circ} 4$ se muestran estos resultados, los que indican que los errores oscilan entre 0,05 y 0,91 mm/día. Los métodos de Turc y de Penman tienen los errores más bajos en todas las estaciones, alejándose entre 0,05 y $0,13 \mathrm{~mm} /$ día del promedio. En el otro extremo, el de Ivanov da los errores más altos en todas las estaciones, particularmente en las costeras y del secano interior, variando entre 0,28 y $0,91 \mathrm{~mm} /$ día. Le sigue a Ivanov, Jensen-Haise (entre 0,27 y 0,50 mm/ día), aunque no en todos los observatorios. Si bien estas tendencias son consistentes, debe señalarse que puede haber cierto sesgo en estos resultados dado que hay estaciones que no contaban con su serie de datos completa.

Se calcularon otros valores que se consideraron como estándares de comparación, obteniéndose resultados muy similares a los ya señalados y que muestran a Penman y Turc con errores máximos de alrededor $1 \mathrm{~mm} /$ día.

El análisis de regresión entre cada uno de los métodos y el valor promedio permitió observar la sobrestimación o subestimación en los valores de ETo que se obtiene con

Figura $\mathrm{N}^{\circ} 2$

Evolución temporal de la ETo ( $\mathrm{mm} /$ día) estimada con los seis métodos seleccionados en la estación Paredones (Pa)

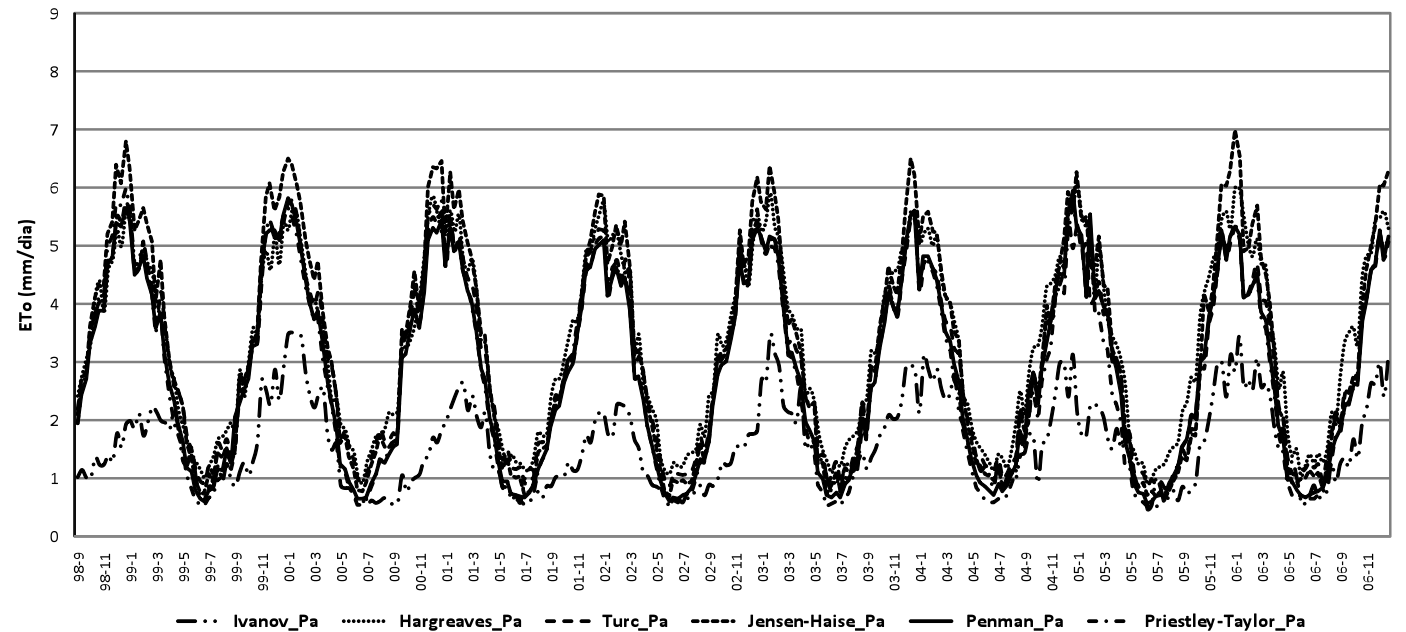

Nota: Valores decadales entre octubre de 1998 y diciembre de 2006.

Fuente: Elaboración propia. 
Figura $\mathrm{N}^{\circ} 3$

Evolución temporal de la ETo ( $\mathrm{mm} /$ día) estimada con los seis métodos seleccionados en la estación Quinta de Tilcoco (QT)

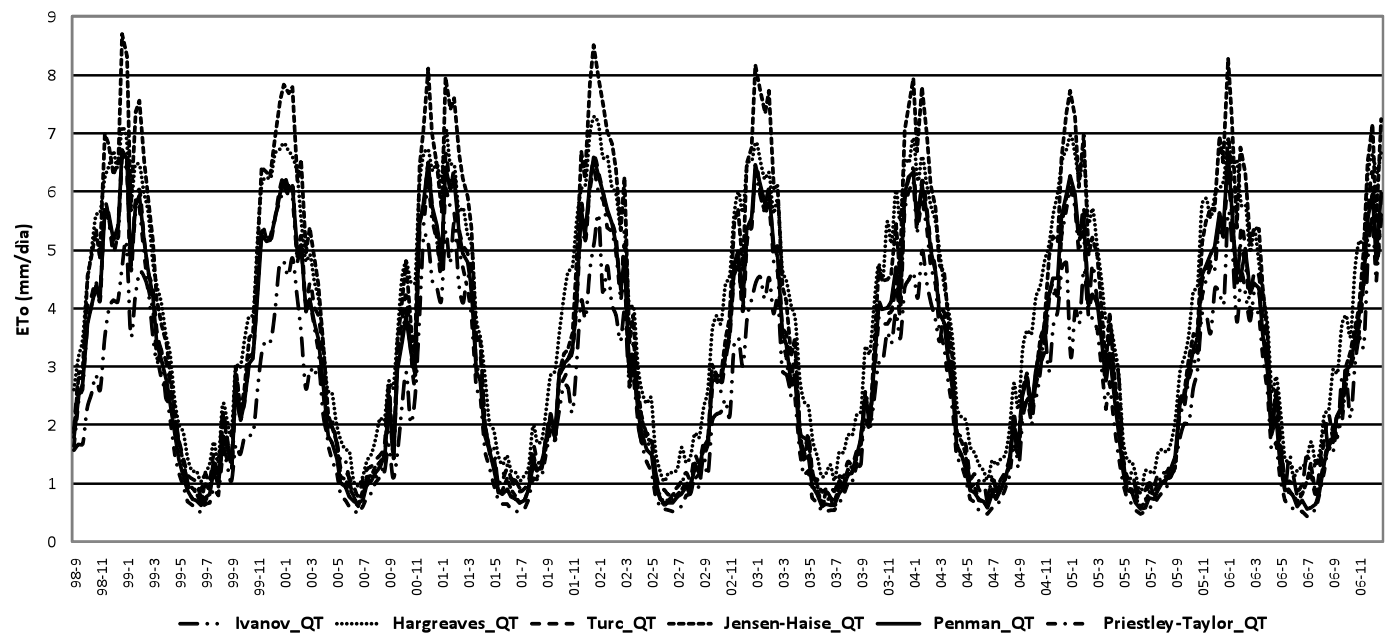

Nota: Valores decadales entre octubre de 1998 y diciembre de 2006.

Fuente: Elaboración propia.

Figura $\mathrm{N}^{\circ} 4$

Error cuadrático medio ( $\mathrm{mm} /$ día) de cada método en relación al valor estándar en los doce observatorios meteorológicos

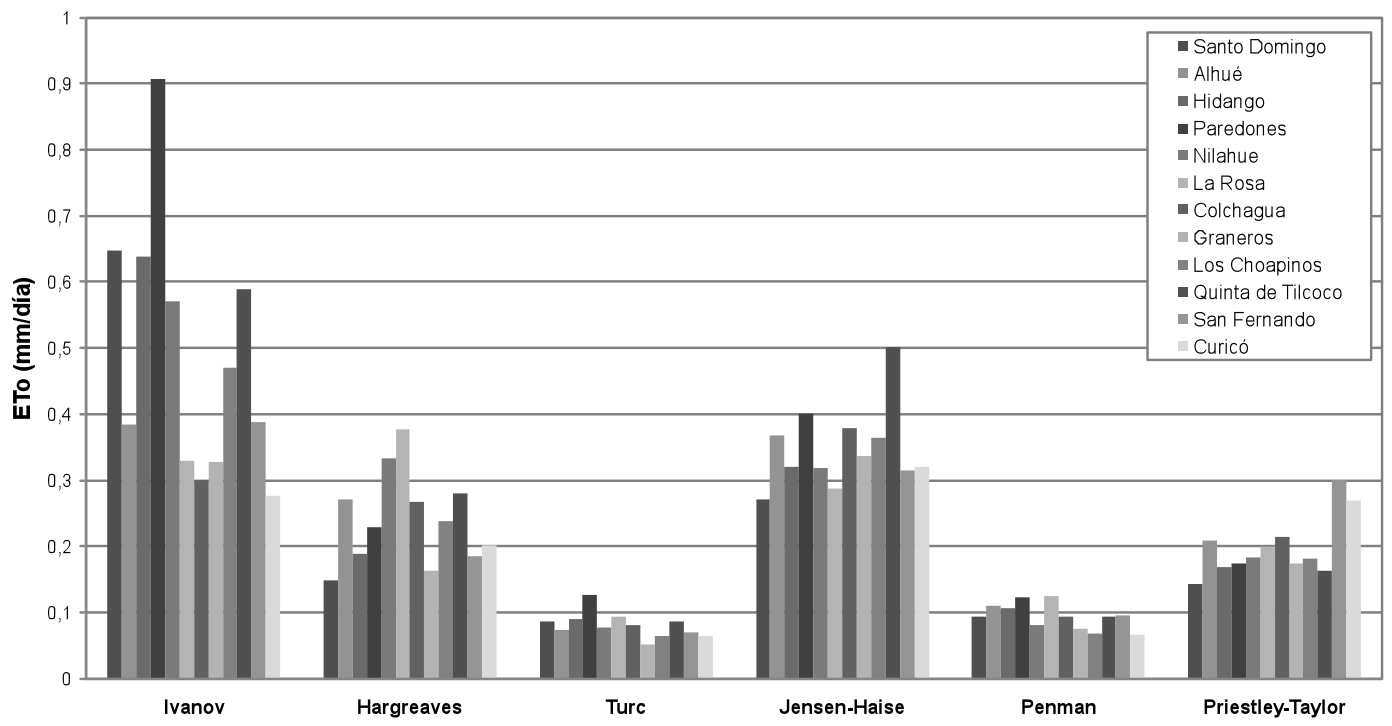

Fuente: Elaboración propia. 
cada método y en cada una de las estaciones utilizadas. Las Figuras $N^{\circ} 5$ y $N^{\circ} 6$ muestran los resultados obtenidos en las estaciones de Paredones y Quinta de Tilcoco, respectivamente. En Quinta de Tilcoco y, en general, en todas las estaciones de interior, hay un mejor ajuste entre los valores, comparado con $\mathrm{Pa}$ redones y las estaciones del secano costero y secano interior. Ello se advierte en la forma de la dispersión, en concreto, en la mayor cercanía de las observaciones a la recta de mínimos cuadrados, lo que, a su vez, se refleja en mayores valores de R cuadrado.

En las dos estaciones de referencia la tendencia de los métodos es análoga. El mejor ajustado al promedio es Penman y en ambos casos con R cuadrado superior a 0,99; luego viene Turc, con valores también superiores a 0,99; le sigue Jensen-Haise, Priestley-Taylor y Hargreaves, $y$, finalmente, Ivanov, que es el que presenta la mayor dispersión de puntos con respecto a la recta y los menores valores de R cuadrado.
Hay métodos que presentan ciertos sesgos en las estimaciones, según la magnitud de los valores de ETo. Como patrones observables en los dos ejemplos que se presentan está la menor precisión del método de Ivanov, en particular, en la estación de Paredones, y la sobrestimación y subestimación del método de Hargreaves, según las magnitudes de ETo. En el caso de esta última ecuación, el patrón común es una leve tendencia a la sobrestimación en los valores medios de ETo y una tendencia a la subestimación en los valores altos, siendo esto más marcado en el caso de Quinta de Tilcoco. También es de destacar que en el método de Priestley-Taylor hay una pérdida progresiva de precisión a medida que la ETo se hace mayor en Paredones, aunque en Quinta de Tilcoco este comportamiento no se aprecia. No obstante esto último, PriestleyTaylor, Jensen-Haise, Turc y Penman presentan un buen ajuste.

Con respecto a la significancia de las diferencias de la ETo estimada, esta se ha

Figura $\mathrm{N}^{\circ} 5$

Modelos de regresión de ETo $(\mathrm{mm} /$ día) entre cada método utilizado respecto al valor promedio. Estación Paredones
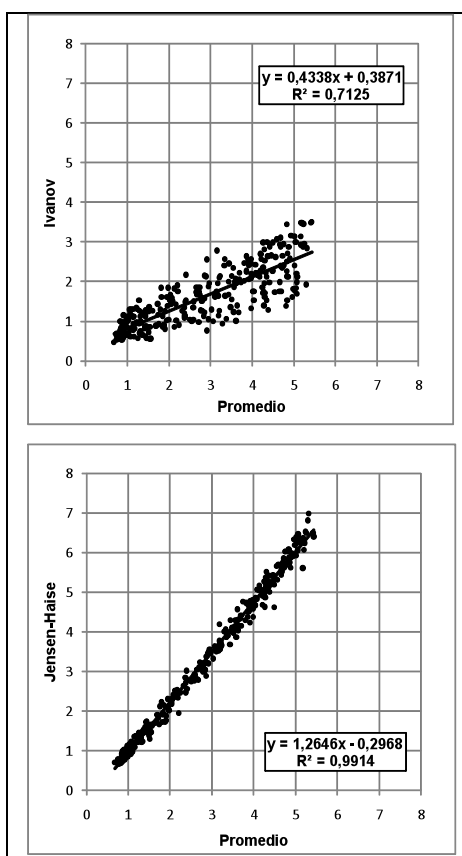

Fuente: Elaboración propia.
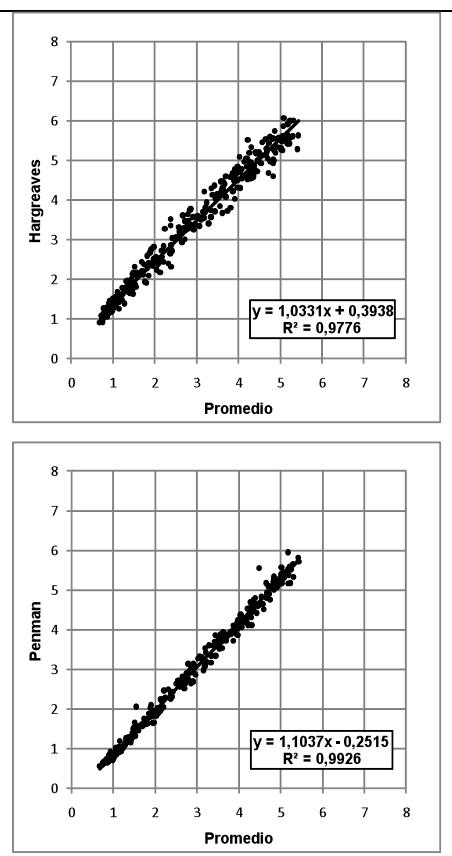
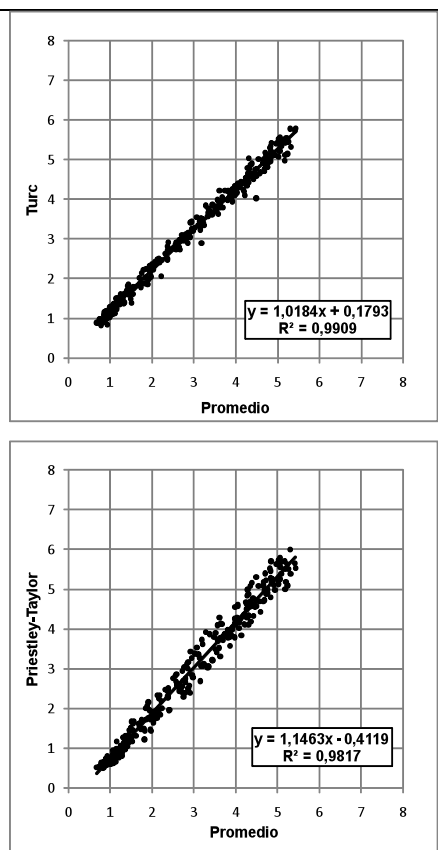
evaluado mediante un análisis de varianza aplicado a todos los métodos empleados por observatorio meteorológico. Los análisis realizados hasta el momento muestran una gran similitud general en las tendencias de los métodos, lo que confirma que todos siguen comportamientos que pueden asignarse a un mismo conjunto de patrones determinantes de su respuesta. Sin embargo, la similitud de tendencias no necesariamente implica una semejanza en las magnitudes de ETo que los métodos están estimando, por lo que resulta de interés averiguar si existen diferencias significativas entre estos valores.

Los valores calculados de $\mathrm{F}$ pueden verse en el Cuadro $N^{\circ}$ 3. De acuerdo a los grados de libertad intra muestras y entre muestras, el valor crítico de $\mathrm{F}$ con un $99 \%$ de certeza es de 2,51 (Ebdon, p. 324), de donde se puede inferir que, al ser en todos los casos el valor de F calculado mayor a 2,51, en todas las estaciones meteorológicas estudiadas se obtienen valores de ETo significativamente diferen-
Cuadro $\mathrm{N}^{\circ} 3$

Cálculo de F para las estaciones seleccionadas

\begin{tabular}{|l|c|}
\hline Estación & Valor F \\
\hline Alhué & 6,91 \\
Colchagua & 4,52 \\
Curicó & 6,82 \\
Graneros & 7,27 \\
Hidango & 8,32 \\
Los Choapinos & 6,61 \\
La Rosa & 6,76 \\
Nilahue & 6,44 \\
Paredones & 24,30 \\
Quinta de Tilcoco & 7,46 \\
Santo Domingo & 28,12 \\
San Fernando & 7,15 \\
\hline
\end{tabular}

Fuente: Elaboración propia.

Figura $\mathrm{N}^{\circ} 6$

Modelos de regresión de ETo ( $\mathrm{mm} /$ día) entre cada método utilizado respecto al valor promedio. Estación Quinta de Tilcoco
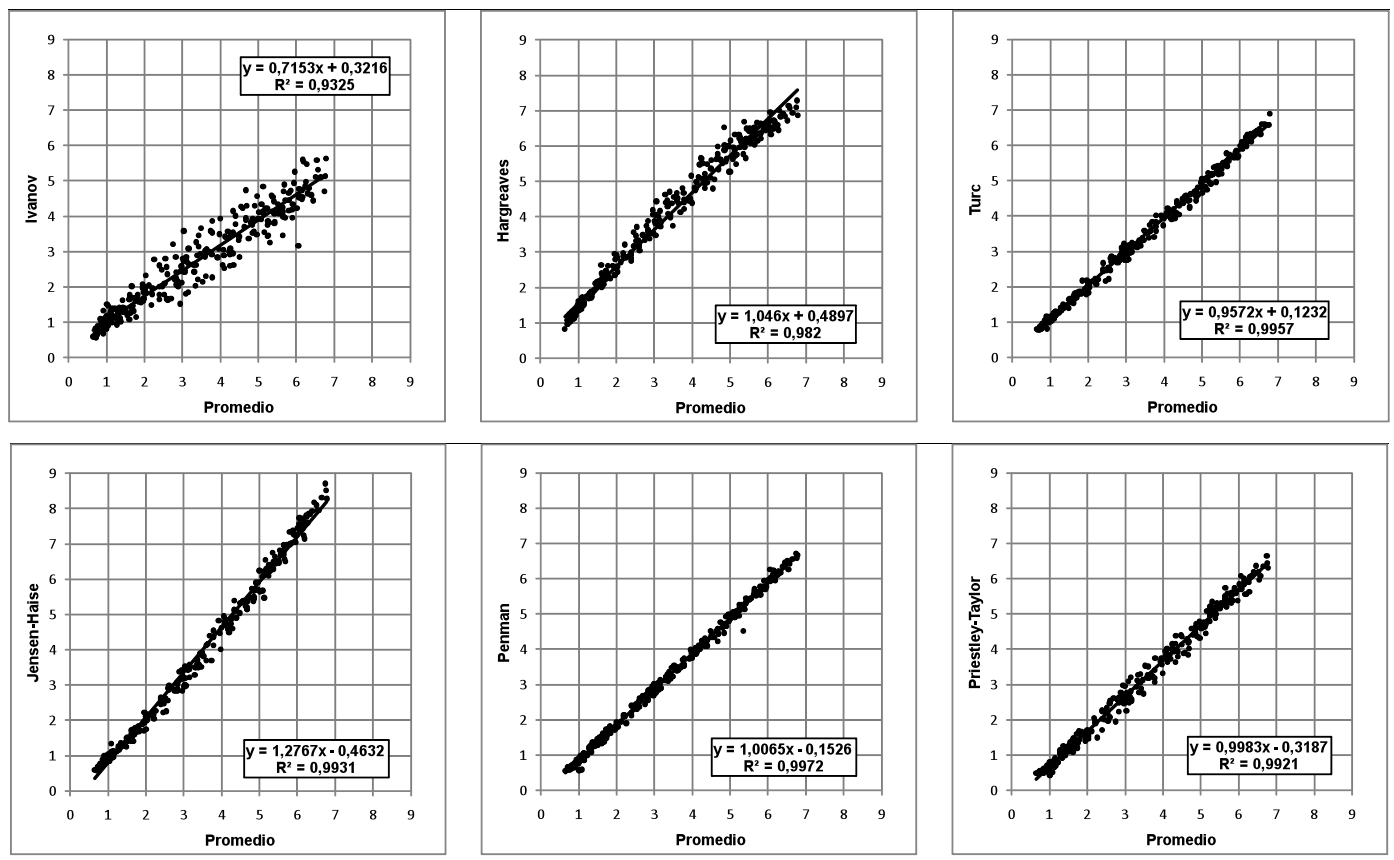

Fuente: Elaboración propia. 
tes en función de los métodos empleados. Los valores mayores de $\mathrm{F}$ tienden a darse en las estaciones costeras o de influencia oceánica, en particular Santo Domingo y Paredones. Un estudio más detallado estación por estación y método por método no entra en los objetivos de este estudio, pero se abordará en trabajos posteriores.

\section{Consideraciones finales}

Los resultados presentados son consistentes con los obtenidos en numerosas investigaciones realizadas en Chile y el mundo. Lo mismo se puede decir si se relacionan con lo obtenido por los autores en una investigación anterior (Sánchez y Carvacho, 2005). El modelo de Turc, basado en la radiación, y el de Penman, en la ecuación de combinación, tienden a ser los más consistentes entre sí y se ajustan muy bien al promedio, en todas las estaciones, revelando consistencia y predictibilidad. En el otro extremo, Ivanov es el que presenta mayor dispersión con respecto a todos los otros métodos. Lamentablemente, no hay muchas referencias que permitan concluir con más fundamentos si estos resultados se condicen con eventuales usos del método en otros ambientes. Al igual que en Temesgen et al. (2005), se confirma en este estudio al método de Hargreaves como una buena alternativa ante la imposibilidad del cálculo de ecuaciones de mayor demanda de datos. Esto considerando que la temperatura es una de las variables de registro más común y que puede ser interpolada, espacial y temporalmente, con mayor seguridad que cualquier otra variable meteorológica.

Es recomendable profundizar en los resultados que entregan los métodos realizando un análisis temporal que permita determinar en qué épocas del año los resultados son diferentes y en qué magnitud, de manera de tener mayor claridad para el uso de los métodos. Por otra parte, se espera también en el futuro integrar en el análisis otras ecuaciones de combinación, recomendadas actualmente, aunque más difíciles de calcular.

A falta de medidas en terreno, resultados más concluyentes podrían ser logrados con la disponibilidad de un mayor número de estaciones meteorológicas y también mejor distribuidas. Esta situación no tiene buenas expectativas en el futuro cercano, si se toma en cuenta que para los últimos años considerados en el estudio, hay menor cantidad de datos disponibles.

\section{Referencias bibliográficas}

ADEBOYE, O. B.; OSUNBITAN, J. A.; ADEKALU, K. O. \& OKUNADE, D. A. Evaluation of FAO-56 Penman-Monteith and temperature based models in estimating reference evapotranspiration using complete and limited data, application to Nigeria. Agricultural Engineering International: the CIGR Ejournal, 2009, vol. XI, № 1291, p. 1-25.

ALLEN, R. G.; PEREIRA, L. S.; RAES, D. y SMITH, M. Evapotranspiración del cultivo. Guías para la determinación de los requerimientos de agua de los cultivos. Roma: Estudio FAO, Riego y Drenaje No 56, 1998.

BAUTISTA, F.; BAUTISTA, D. \& DELGADO-CARRANZA, C. Calibration of the equations of Hargreaves and Thornthwaite to estimate the potential evapotranspiration in semi-arid and subhumid tropical climates for regional applications. Atmósfera, 2009, vol. $22, N^{\circ} 4$, p. 333-348.

BURMAN, R. \& POCHOP, L. O. Evaporation, Evapotranspiration and Climatic Data. Developments in Atmospheric Science, 22. Amsterdam: Elsevier, 1994.

CHEMIN, Y. Evapotranspiration of crops by remote sensing using the energy balance based algorithms. In: $1^{\text {st }}$ International Yellow River Forum on River Basin Management. Zhengzhou: International Yellow River Forum, 2003, p. 76-85.

COMISIÓN NACIONAL DE RIEGO. Cálculo y cartografía de la evapotranspiración potencial en Chile. Santiago: Comisión Nacional de Riego, 1997.

DOORENBOS, J. y PRUITT, W.O. Las necesidades de agua de los cultivos. Roma: Estudio FAO, Riego y Drenaje № 24, 1977.

DOUGLAS, E. M.; JACOBS, J. M.; SUMNER, D. M. \& RAY, R. I. A comparison 
of models for estimating potential evapotranspiration for Florida land cover types. Journal of Hydrology, 2009, № 373, p. 366-376.

DROOGERS, P. \& ALLEN, R.G. Estimating reference evapotranspiration under inaccurate data conditions. Irrigation and Drainage Systems, 2002, 16, p. 33-45.

EBDON, D. Estadística para Geógrafos. Barcelona: Oikos-Tau, 1982.

FERNÁNDEZ, F. Manual de climatología aplicada. Clima, medio ambiente, planificación. Editorial Síntesis. Madrid: Colección Espacios y Sociedades, Serie Mayor No 2, 1995.

HARGREAVES, G. H. Defining and using reference evapotranspiration. Journal of Irrigation and Drainage Engineering, ASCE, 1994, vol. 120, No6, p. 1132-1139.

INSTITUTO DE INVESTIGACIONES AGROPECUARIAS. Mapa Agroclimático de Chile. Santiago: Instituto de Investigaciones Agropecuarias, 1989.

JABULANI, J. Evaluation of the potential of using the modified JensenHaise model as an irrigation scheduling technique in Zimbabwe. Electronic Journal of Environmental, Agricultural and Food Chemistry, EJEAFChe, 2008, vol. 7, № 14, p. 2771-2778.

JENSEN, D. T.; HARGREAVES, F. H.; TEMESGEN, B. \& ALLEN, R. G. Computation of ETo under nonideal conditions. Journal of Irrigation and Drainage Engineering, ASCE, 1997, september/october, p. 394-400.

JENSEN, M. E.; BURMAN, R. D. \& ALLEN, R. G. (eds.). Evapotranspiration and irrigation water requirements. Manuals and Reports on Engineering Practice, $N^{\circ} 70$. New York: Committee on Irrigation Water Requirements of the Irrigation and Drainage Division of the American Society of Civil Engineers, ASCE, 1990.

LÓPEZ-MORENO, J. L.; HESS, T. M. \& WHITE, M. Estimation of reference evapotranspiration in a mountainous mediterranean site using the PenmanMonteith equation with limited meteorological Data. Pirineos, 2009, No 164, p. 7-31.

MCKENNEY, M. S. \& ROSENBERG, N. J. Sensitivity of some potential evapotranspiration estimation methods to climate change. Agricultural and Forest Meteorology, 1993, vol. 64, p. 81-110.

MERLET, H. A. Evapotranspiración potencial y necesidades netas de agua de riego en Chile. Tesis Licenciado en Ciencias Agrícolas. Santiago, Escuela de Agronomía, Facultad de Ciencias Agrarias y Forestales, Universidad de Chile, Chile, 1986.

MERLET, H. y SANTIBÁÑEZ, F. Evaluación y cartografía de la evapotranspiración potencial en la zona de climas mediterráneos de Chile. Boletín Técnico, Facultad de Ciencias Agrarias y Forestales, Universidad de Chile, 1989, № 48, p. 27-50.

PEREIRA, D. D.; YANAGI, S. D. M.; DE MELLO, C. R.; DA SILVA, A. M. \& DA SILVA, L. A. Performance of the reference evapotranspiration estimating methods for the Mantequeira range region, MG, Brazil. Ciencia Rural, 2009, vol. 39, No 9, p. 24882493.

ROSENBERG, N. J.; BLAD, B. L. \& VERMA, S. B. Microclimate, the biological environment. New York: John Wiley \& Sons, second edition, 1983.

SÁNCHEZ, M. Estimación de evapotranspiración a través de datos meteorológicos e imágenes de satélite. Tesis doctoral. Departamento de Geografía, Universidad de Alcalá de Henares, España, 1999.

SÁNCHEZ, M. Características y apreciaciones generales de los métodos de medida y estimación de evapotranspiración. Revista de Geografía Norte Grande, 2000, № 27, p. 27-36.

SÁNCHEZ, M. Métodos de estimación de evapotranspiración utilizados en Chile. Revista de Geografía Norte Grande, 2001, No 28, p. 3-10. 
SÁNCHEZ, M. I. Métodos para el estudio de la evaporación y evapotranspiración. Cuadernos Técnicos de la Sociedad Española de Geomorfología No 3. Logroño: Geoforma Ediciones, 1992.

SÁNCHEZ, M. y CARVACHO, L. Comparación de diez métodos de estimación de evapotranspiración potencial. Caso de estudio: VI Región del Libertador General Bernardo O'Higgins. Anales de la Sociedad Chilena de Ciencias Geográficas, 2005, p. 129-142.

SANTIBÁÑEZ, F.; PARADA, M. y ULRIKSEN, P. Perspectivas de desarrollo de los recursos de la VII Región. Distritos agroclimáticos. Santiago: Instituto Nacional de Investigación de Recursos Naturales, IREN-CORFO, Publicación 25, 1979.

SHI, T.; GUAN, D.; WANG, A.; WU, A.; JIN, C. \& HAN S. Comparison of three models to estimate evapotranspiration for a temperate mixed forest. Hydrological Processes, 2008, № 22, p. 3431-3443.

SULEIMAN, A. A. \& HOOGENBOOM, G. Comparison of Priestley-Taylor and FAO-56 Penman-Monteith for daily reference evapotranspiration. Estimation in Georgia. Journal of Irrigation and Drainage Engineering, ASCE, 2007, march/april, p. 175182.
TEMESGEN, B.; ECHING, S.; DAVIDOFF, B. \& FRAME, K. Comparison of some reference evapotranspiration equations for California. Journal of Irrigation and Drainage Engineering, ASCE, 2005, january/february, p. 73-84.

VALENZUELA, A. y FERREIRA, V. Variación de la evapotranspiración potencial en Chile. Agro-Ciencia, 1985, vol. 1, № 1, p. 15-21.

VEGA, E. J. y JARA, J. C. Estimación de la evapotranspiración de referencia para dos zonas (costa y región andina) del Ecuador. Engenharia Agrícola, 2009, vol. 9, № 3, p. 390-403.

XU, C. Y. \& SINGH, V. P. Dependence of evaporation on meteorological variables at different time-scales and intercomparison of estimation methods. Hydrological Processes, 1998, No 12, p. 429-442.

$X U$, C. Y. \& SINGH, V. P. Evaluation and generalization of temperature-based methods for calculating evaporation. Hydrological Processes, 2001, No 13, p. 305-319.

$X U$, C. Y. \& SINGH, V. P. Cross comparison of empirical equations for calculating potential evapotranspiration with data from Switzerland. Water Resources Management, 2002, № 16, p. 197-219. 